\title{
A CONVERGENCE ANALYSIS OF REGULARIZATION BY DISCRETIZATION IN PREIMAGE SPACE
}

\author{
BARBARA KALTENBACHER AND JONAS OFFTERMATT
}

\begin{abstract}
In this paper we investigate the regularizing properties of discretization in preimage space for linear and nonlinear ill-posed operator equations with noisy data. We propose to choose the discretization level, that acts as a regularization parameter in this context, by a discrepancy principle. While general convergence has been shown not to hold, we provide convergence results under appropriate conditions on the exact solution.
\end{abstract}

\section{INTRODUCTION}

A wide range of inverse problems can be formulated as nonlinear ill-posed operator equations,

$$
F(x)=y
$$

with a forward operator $F: D(\subseteq X) \rightarrow Y$, mapping between Hilbert spaces $X$ and $Y$.

Since typically $F^{-1}$ is not continuous and only noisy data $y^{\delta}$ with some noise level $\delta$ in

$$
\left\|y-y^{\delta}\right\| \leq \delta
$$

are available, regularization has to be applied (see, e.g., 11, 4, 6, 17, 20, 21, 22, 25, 27, 31, 33, 34, ).

Motivated by the fact that on one hand, in practice, very often inverse problems are just discretized and then solved, on the other hand this discretization itself can be expected to have a regularizing effect, we investigate convergence in the sense of a regularization method for discretization in preimage space. For this purpose we consider a sequence $\left(X_{n}\right)_{n \in \mathbb{N}}$ of finite dimensional subspaces of the preimage space $X_{n} \subseteq X$, along with the corresponding orthogonal projections $P_{n}:=\operatorname{Proj}_{X_{n}}$, and assume that $\left\|\left(I-P_{n}\right) x^{\dagger}\right\| \rightarrow 0$ as $n \rightarrow \infty$. (The latter is the case, e.g., if $X_{n+1} \subseteq X_{n}$ and $\overline{\bigcup_{n \in \mathbb{N}} X_{n}}=X$.) Therewith we define a regularized approximation as a solution of the finite dimensional minimization problem

$$
x_{n}^{\delta} \in \operatorname{argmin}\left\{\left\|F\left(z_{n}\right)-y^{\delta}\right\|^{2}: z_{n} \in D \cap X_{n}\right\},
$$

an approach which is also often called the least squares projection. The discretization level $n$ has to be chosen appropriately in order to balance between the approximation error, that decays as $n \rightarrow \infty$ and the noise propagation, that becomes

Received by the editor April 10, 2011 and, in revised form, June 26, 2011.

2010 Mathematics Subject Classification. Primary 65J20, Secondary 65M32.

Support by the German Science Foundation (DFG) within the Cluster of Excellence in Simulation Technology (EXC 310/1) at the University of Stuttgart is gratefully acknowledged.

(C) 2012 American Mathematical Society Reverts to public domain 28 years from publication 
stronger for larger $n$. Here, we determine $n_{*}$ by the discrepancy principle

$$
n_{*}=\min \left\{n \in \mathbb{N}:\left\|F\left(x_{n}^{\delta}\right)-y^{\delta}\right\| \leq \tau \delta\right\}
$$

(with $\tau>1$ in the linear case, and $\tau>\frac{1+\eta}{1-\eta}$, with $\eta \in(0,1)$ as in (1.5) below in the nonlinear case). A very efficient approach especially in the context of nonlinear problems is to apply a multilevel strategy for the computation of the regularized solution, i.e., to start on the coarsest level $n=1$ of projection and successively compute the solution on level $n$ making use of information on the solution on level $n-1$. When doing so, the discrepancy principle just acts as a stopping rule for this multilevel iteration.

Regularization by discretization in image space (also called the dual least squares method or self regularization) has been well investigated in the literature (see, e.g., [26, 28, 32] for the linear case, as well as [10, 12] for the nonlinear case) and can be shown to be generically convergent. However, regularization by discetization in preimage space (called least squares method in [26]) is used more often in practice due its often much easier implementation. Additionally, it is of particular interest due to the possibility of using problem adapted ansatz functions for the inverse problem solution, as well as the above mentioned rather straightforward multilevel strategy. Previous results in the literature on this approach for linear ill-posed problems [7, 8, 23], and especially a counterexample by Seidman [30] indicate, that convergence cannot occur for general $x^{\dagger} \in X$ but only under special assumptions on the solution. Here we formulate a convergence condition that assumes sufficiently fast convergence of the approximation error in image space to compensate for the growth of the norm of the inverse of the projected forward operator. This sufficiently fast convergence of the approximation error in its turn will be implied by a sufficiently strong source condition and hence is satisfied for sufficiently regular solutions, along with appropriate approximation properties of the ansatz spaces $X_{n}$.

We wish to mention that the regularizing effect of discretization has been studied in a very general setting with possible discretization of both preimage and image space, e.g., in Chapter 3 of 20] and in 24] for the linear case. The latter also provides results on convergence and convergence rates for noisy data with an a priori and a Lepskii type a posteriori discretization level choice.

Our new results especially pertain to taking into account noisy data by means of a discrepancy principle type a posteriori discretization level choice, as well as to extension to the nonlinear situation.

It is well-known that a convergence analysis for nonlinear ill-posed problems requires some assumption on the structure of the nonlinearity. Here we will assume that the often used Scherzer condition (also called tangential cone condition, cf. [29]) holds:

$$
\left\|F(x)-F(\bar{x})-F^{\prime}(x)(x-\bar{x})\right\| \leq \eta\|F(x)-F(\bar{x})\| \quad \forall x, \bar{x} \in D
$$

with $\eta \in(0,1)$, which implies

$$
\|F(x)-F(\bar{x})\| \leq \frac{1}{1-\eta}\left\|F^{\prime}(x)(x-\bar{x})\right\|,\left\|F^{\prime}(x)(x-\bar{x})\right\| \leq(1+\eta)\|F(x)-F(\bar{x})\| .
$$

Note that for this purpose $F^{\prime}(x)$ need not necessarily be a Fréchet derivative. It suffices to have a bounded linear operator called $F^{\prime}(x)$ mapping between $X$ and $Y$ and such that (1.5) holds. 
In what follows we will assume that an exact solution $x^{\dagger}$ of (1.1) exists, i.e., $F\left(x^{\dagger}\right)=y$. Moreover, to simplify the exposition we assume that

$$
\forall x \in D: \quad \mathcal{N}\left(F^{\prime}(x)\right)=\{0\},
$$

which implies uniqueness of the solution to $F(x)=y$ in the linear case and by (1.5) also in the nonlinear case: From $0=\|F(x)-F(\tilde{x})\| \geq \frac{1}{1+\eta}\left\|F^{\prime}(x)(x-\tilde{x})\right\|$ it follows that $x-\tilde{x} \in \mathcal{N}\left(F^{\prime}(x)\right)=\{0\}$. Note, however, that most of our results can be generalized to operators with nontrivial nullspaces.

The remainder of this paper is organized as follows. In Section 2, we first provide a convergence analysis for the linear case, whereas the general nonlinear situation is treated in Section 3 For the latter generalization we follow two approaches, namely a variational formulation and an operator equation formulation. Numerical experiments in Section 4 illustrate the results of our convergence analysis.

\section{THE LINEAR CASE}

In this section we first consider the linear case $F=T \in L(X, Y)$, so that (1.1) becomes

$$
T x=y .
$$

The first order necessary condition for a minimizer of (1.3), i.e., of

$$
\min _{z_{n} \in X_{n}}\left\|T z_{n}-y^{\delta}\right\|^{2}=\min _{z_{n} \in X_{n}}\left\|T P_{n} z_{n}-y^{\delta}\right\|^{2}
$$

implies

$$
\left(T P_{n}\right)^{*}\left(T P_{n} z_{n}-y^{\delta}\right)=0
$$

hence

$$
T P_{n} z_{n}-y^{\delta} \in \mathcal{N}\left(\left(T P_{n}\right)^{*}\right)=\mathcal{R}\left(T P_{n}\right)^{\perp}=Y_{n}^{\perp},
$$

i.e.,

$$
Q_{n}\left(T P_{n} x_{n}^{\delta}-y^{\delta}\right)=0
$$

with the orthogonal projections $Q_{n}:=\operatorname{Proj}_{Y_{n}}$, and the subspaces $Y_{n}:=T X_{n} \subseteq Y$; see also [20, 21, 24] for a convergence analysis of $x_{n}^{\delta}$ defined by (2.4) with general $Y_{n}$, i.e., not necessarily $Y_{n}=T X_{n}$.

\subsubsection{Well-definedness.}

Lemma 2.1. The index $n_{*}$ according to the discrepancy principle (1.4) with $\tau>1$ is well defined.

Proof. The residual can be represented as follows:

$$
\begin{aligned}
T x_{n}^{\delta}-y^{\delta} & =\left(I-Q_{n}\right)\left(T x_{n}^{\delta}-y^{\delta}\right)=-\left(I-Q_{n}\right) y^{\delta} \\
& =-\left(I-Q_{n}\right) T x^{\dagger}+\left(I-Q_{n}\right)\left(y-y^{\delta}\right) \\
& =-\left(I-Q_{n}\right) T\left(I-P_{n}\right) x^{\dagger}+\left(I-Q_{n}\right)\left(y-y^{\delta}\right)
\end{aligned}
$$

where we have used $T x_{n}^{\delta} \in Y_{n}$ in the second equality and $T P_{n} x^{\dagger} \in Y_{n}$ in the fourth equality. From (2.5), and $\left\|I-Q_{n}\right\| \leq 1$ we get

$$
\left\|T x_{n}^{\delta}-y^{\delta}\right\| \leq\|T\| \underbrace{\left\|\left(I-P_{n}\right) x^{\dagger}\right\|}_{\rightarrow 0 \text { as } n \rightarrow \infty}+\delta,
$$

hence $\left\|T x_{n}^{\delta}-y^{\delta}\right\| \leq \tau \delta$ for $n$ sufficiently large. 
Denote

$$
\begin{gathered}
\alpha_{n}:=\left\|\left(I-P_{n}\right) x^{\dagger}\right\|, \quad \beta_{n}:=\left\|T\left(I-P_{n}\right) x^{\dagger}\right\| \\
\gamma_{n}:=\inf _{\substack{z_{n} \in X_{n} \\
z_{n} \neq 0}} \frac{\left\|T z_{n}\right\|}{\left\|z_{n}\right\|}=\inf _{z_{n} \in X_{n}} \frac{\left\|T z_{n}\right\|}{\left\|\left(T P_{n}\right)^{\dagger} T P_{n} z_{n}\right\|} \\
=\inf _{\substack{z_{n} \neq 0 \\
w_{n} \in T X_{n} \\
w_{n} \neq 0}} \frac{\left\|w_{n}\right\|}{\left\|\left(T P_{n}\right)^{\dagger} w_{n}\right\|}=\frac{1}{\left\|\left(T P_{n}\right)^{\dagger}\right\|},
\end{gathered}
$$

where we have used the fact that by $\mathcal{N}(T)=\{0\}$ we have

$$
\mathcal{N}\left(T P_{n}\right)^{\perp}=\overline{\mathcal{R}\left(P_{n} T^{*}\right)}=P_{n} \overline{\mathcal{R}\left(T^{*}\right)}=P_{n} \mathcal{N}(T)^{\perp}=P_{n} X=X_{n} .
$$

In the following proofs we will use the error decomposition

$$
\begin{aligned}
x_{n}^{\delta}-x^{\dagger} & =x_{n}^{\delta}-P_{n} x^{\dagger}-\left(I-P_{n}\right) x^{\dagger} \\
& =\left(T P_{n}\right)^{\dagger} T P_{n}\left(x_{n}^{\delta}-P_{n} x^{\dagger}\right)-\left(I-P_{n}\right) x^{\dagger} \\
& =\left(T P_{n}\right)^{\dagger}\left(y^{\delta}-T P_{n} x^{\dagger}\right)-\left(I-P_{n}\right) x^{\dagger} \\
& =\left(T P_{n}\right)^{\dagger}\left(y^{\delta}-y\right)+\left(T P_{n}\right)^{\dagger} T\left(I-P_{n}\right) x^{\dagger}-\left(I-P_{n}\right) x^{\dagger},
\end{aligned}
$$

where we have used (2.4) and (2.8).

\subsubsection{Stability for fixed discretization level.}

Proposition 2.2. Let $n$ be fixed and let $\left(y^{k}\right)_{k \in \mathbb{N}}$ be a sequence converging to $y^{\delta}$ : $\left\|y^{k}-y^{\delta}\right\| \rightarrow 0$ as $k \rightarrow \infty$ and denote by $x_{n}^{k}$ the corresponding regularized solutions according to (2.4) with $y^{\delta}$ replaced by $y^{k}$.

Then

$$
\left\|x_{n}^{k}-x_{n}^{\delta}\right\| \rightarrow 0 \quad \text { as } k \rightarrow \infty
$$

Proof. From (2.9) we obtain

$$
\left\|x_{n}^{k}-x_{n}^{\delta}\right\|=\left\|\left(T P_{n}\right)^{\dagger}\left(y^{k}-y^{\delta}\right)\right\| \leq\left\|\left(T P_{n}\right)^{\dagger}\right\|\left\|y^{k}-y^{\delta}\right\| \rightarrow 0
$$

as $k \rightarrow \infty$.

2.1.3. Convergence.

Theorem 2.3. If

$$
\frac{\beta_{n}}{\gamma_{n}} \rightarrow 0, \quad \frac{\beta_{n-1}}{\gamma_{n}} \rightarrow 0, \quad \alpha_{n} \rightarrow 0, \quad \text { as } n \rightarrow \infty,
$$

then for $n_{*}=n_{*}(\delta)$ according to (1.4),

$$
\left\|x_{n_{*}(\delta)}^{\delta}-x^{\dagger}\right\| \rightarrow 0 \text { as } \delta \rightarrow 0 \text {. }
$$

Proof. From (2.9) we get

$$
\left\|x_{n}^{\delta}-x^{\dagger}\right\| \leq \frac{\delta}{\gamma_{n}}+\frac{\beta_{n}}{\gamma_{n}}+\alpha_{n} .
$$

Let $\delta_{m}$ be an arbitrary sequence converging to zero and $y_{m}$ a sequence of data satisfying $\left\|y_{m}-y\right\| \leq \delta_{m}$. We denote by $n_{m}$ the corresponding index according to the discrepancy principle and by $x_{n_{m}}^{\delta_{m}}$ the approximation on level $n_{m}$ according to (2.4) with $y^{\delta}$ replaced by $y_{m}$.

Consider first the case that $n_{m} \rightarrow \infty$ as $m \rightarrow \infty$. Then according to (2.5) we can estimate as follows:

$$
\tau \delta_{m} \leq\left\|T x_{n_{m}-1}^{\delta_{m}}-y_{m}\right\| \leq\left\|\left(I-Q_{n_{m}-1}\right) T\left(I-P_{n_{m}-1}\right) x^{\dagger}\right\|+\delta_{m},
$$


hence

$$
\frac{\delta_{m}}{\gamma_{n_{m}}} \leq \frac{1}{\tau-1} \frac{\beta_{n_{m}-1}}{\gamma_{n_{m}}}
$$

so by (2.11) we get

as $m \rightarrow \infty$.

$$
\left\|x_{n_{m}}^{\delta_{m}}-x^{\dagger}\right\| \leq \frac{1}{\tau-1} \frac{\beta_{n_{m}-1}}{\gamma_{n_{m}}}+\frac{\beta_{n_{m}}}{\gamma_{n_{m}}}+\alpha_{n_{m}} \rightarrow 0
$$

In the alternative case that $\left(n_{m}\right)$ has a finite accumulation point there exists an $N \in \mathbb{N}$ and a subsequence $\left(m_{k}\right)_{k \in \mathbb{N}}$ such that

$$
\forall k \in \mathbb{N}: \quad n_{m_{k}}=N \text {. }
$$

From (2.5) we get

$$
\tau \delta_{m_{k}} \geq\left\|T x_{n_{m_{k}}}^{\delta_{m_{k}}}-y_{m_{k}}\right\| \geq\left\|\left(I-Q_{N}\right) T\left(I-P_{N}\right) x^{\dagger}\right\|-\delta_{m_{k}},
$$

hence

$$
(\tau+1) \delta_{m_{k}} \geq\left\|\left(I-Q_{N}\right) T\left(I-P_{N}\right) x^{\dagger}\right\|
$$

which, with $k \rightarrow \infty$, implies

$$
\left(I-Q_{N}\right) T x^{\dagger}=\left(I-Q_{N}\right) T\left(I-P_{N}\right) x^{\dagger}=0,
$$

i.e., $T x^{\dagger} \in T X_{N}$, i.e., $\exists z_{N} \in X_{N}: T x^{\dagger}=T z_{N}$, i.e., $\exists z_{N} \in X_{N}: x^{\dagger}-z_{N} \in$ $\mathcal{N}(T)=\{0\}$, i.e., $x^{\dagger} \in X_{N}$, i.e, $\left(I-P_{N}\right) x^{\dagger}=0$, hence by (2.11)

as $k \rightarrow \infty$.

$$
\left\|x_{n_{m_{k}}}^{\delta_{m_{k}}}-x^{\dagger}\right\| \leq \frac{\delta_{m_{k}}}{\gamma_{N}} \rightarrow 0
$$

Remark 2.4. Note that the convergence conditions of this theorem imply the convergence criterion $\lim \sup _{n \rightarrow \infty}\left\|x_{n}\right\| \leq\left\|x^{\dagger}\right\|$ for exact data from [7], since

$$
\begin{aligned}
\left\|\left(T P_{n}\right)^{\dagger} y\right\| & \leq\left\|\left(T P_{n}\right)^{\dagger} T P_{n} x^{\dagger}\right\|+\left\|\left(T P_{n}\right)^{\dagger} T\left(I-P_{n}\right) x^{\dagger}\right\| \\
& \leq\left\|Q_{n} x^{\dagger}\right\|+\left\|\left(T P_{n}\right)^{\dagger}\right\|\left\|T\left(I-P_{n}\right) x^{\dagger}\right\| .
\end{aligned}
$$

The difference to the convergence results from Chapter 3 in 20 is that we consider convergence not for all $x^{\dagger} \in X$ but for a particular $x^{\dagger}$ that might have higher regularity than typical elements of $X$ so that divergence to infinity of the factor $\frac{1}{\gamma_{n}}$ due to unboundedness of $T^{\dagger}$ might indeed be compensated by sufficiently fast convergence of the approximation error $\beta_{n}$.

Indeed, note that $\left\|T\left(I-P_{n}\right) x^{\dagger}\right\|$ is likely to go to zero at a faster rate than $\left\|\left(I-P_{n}\right) x^{\dagger}\right\|$, since $T$ typically has a smoothing property. Under an additional source condition,

$$
x^{\dagger}=\left(T^{*} T\right)^{\nu} w
$$

for some index $\nu>0$ and some $w \in X$ the rate of convergence of $\beta_{n}$ to zero will still be improved: If the smoothing property of $T$ can be quantified via boundedness as a mapping in a scale of spaces:

$$
\left\|\left(T^{*} T\right)^{\nu}\right\|_{X \rightarrow X^{\nu}} \leq C_{\nu}
$$

and the approximation property of $X_{n}$ scales according to

$$
\left\|I-P_{n-1}\right\|_{X^{\nu} \rightarrow X} \leq f_{\nu}(n), \quad\left\|I-P_{n-1}\right\|_{X^{1 / 2} \rightarrow X} \leq f_{1 / 2}(n),
$$


(one might think of $X_{n}$ as being finite element spaces with mesh sizes $h=\frac{1}{n}$ and of $X^{\nu}$ being Sobolev spaces of order $s=s(\nu)$ ), then

$$
\begin{aligned}
\left\|T\left(I-P_{n-1}\right) x^{\dagger}\right\| & =\left\|\left(T^{*} T\right)^{1 / 2}\left(I-P_{n-1}\right)^{2}\left(T^{*} T\right)^{\nu} w\right\| \\
& \leq\left\|\left(I-P_{n-1}\right)\left(T^{*} T\right)^{1 / 2}\right\|\left\|\left(I-P_{n-1}\right)\left(T^{*} T\right)^{\nu}\right\|\|w\| \\
& \leq f_{\nu}(n) f_{1 / 2}(n)\|w\| .
\end{aligned}
$$

Typically, for sufficiently good approximation spaces (in the context of finite elements these will have to be of sufficiently high order) the decay of $f_{\nu}(n)$ will be faster for larger $\nu$. On the other hand, $\gamma_{n}$ is independent of the solution, hence the product $f_{\nu}(n) f_{1 / 2}(n) \frac{1}{\gamma_{n}}$ will tend to zero for $\nu$ sufficiently large, i.e., for sufficiently smooth solutions.

Note the necessity of considering a particular solution instead of norm estimates in view of the fact that, e.g., in case of nested spaces $X_{n-1} \subseteq X_{n}$, we have

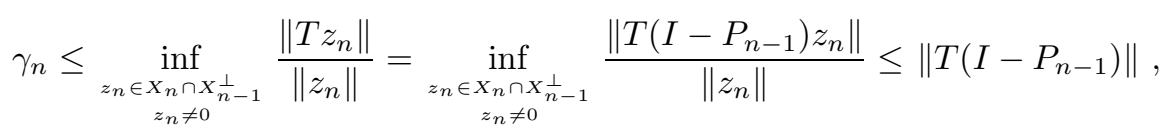

so estimating $\beta_{n-1} \leq\left\|T\left(I-P_{n-1}\right)\right\|\left\|x^{\dagger}\right\|$ would not enable (2.10).

Expressing a possible rate of convergence in (2.10) in terms of $\tilde{\beta}_{n}:=\|(I-$ $\left.Q_{n}\right) T\left(I-P_{n}\right) x^{\dagger} \|$, we can easily deduce a convergence rate result in terms of $\delta$.

Corollary 2.5. Let, with some functions $g_{1}, g_{2}, g_{3}: \mathbb{R}^{+} \rightarrow \mathbb{R}^{+}$satisfying

$$
g_{i}(0)=0, g_{i} \text { monotonically increasing }
$$

and

$$
\forall C>1 \exists \bar{C}(C)>0 \forall \lambda>0: g_{i}(C \lambda) \leq \bar{C}(C) g_{i}(\lambda)
$$

for $i=1,2,3$, the following rates hold in (2.10):

$$
\frac{\beta_{n}}{\gamma_{n}}=O\left(g_{1}\left(\tilde{\beta}_{n}\right)\right), \quad \frac{\tilde{\beta}_{n-1}}{\gamma_{n}}=O\left(g_{2}\left(\tilde{\beta}_{n}\right)\right), \quad \alpha_{n}=O\left(g_{3}\left(\tilde{\beta}_{n}\right)\right), \quad \text { as } n \rightarrow \infty .
$$

Then

$$
\left\|x_{n_{*}(\delta)}^{\delta}-x^{\dagger}\right\|=O\left(\max \left\{g_{1}(\delta), g_{2}(\delta), g_{3}(\delta)\right\}\right) \quad \text { as } \delta \rightarrow 0 .
$$

Proof. Analogously to (2.13), (2.14) we get

$$
\frac{\delta}{\gamma_{n_{*}}} \leq \frac{1}{\tau-1} \frac{\tilde{\beta}_{n_{*}-1}}{\gamma_{n_{*}}}, \quad(\tau+1) \delta \geq \tilde{\beta}_{n_{*}} .
$$

Inserting this and the rates assumptions (2.18) into (2.11), we directly get the assertion.

Remark 2.6. Typical functions satisfying (2.16), (2.17) are $\sigma \mapsto \sigma^{\kappa}, \sigma \mapsto|\ln \sigma|^{-q}$ with $\kappa, q>0$.

Consider the special case of $T$ being compact and $X_{n}=\operatorname{span}\left\{u_{1}, \ldots, u_{n}\right\}$, where $\left(\sigma_{j} ; u_{j}, v_{j}\right)_{j \in \mathbb{N}}$ is a singular system for $T$ (i.e., the method reduces to truncated singular value decomposition). Then a source condition of the form $x^{\dagger}=f\left(\left(T^{*} T\right)\right) w$, 
with an index function $f$, (i.e., $f(0)=0, f$ monotonically increasing) implies

$$
\begin{aligned}
\tilde{\beta}_{n} & =\beta_{n}=\left(\sum_{j=n+1}^{\infty} \sigma_{j}^{2} f\left(\sigma_{j}^{2}\right)^{2}\left\langle w, u_{j}\right\rangle^{2}\right)^{1 / 2}, \\
\gamma_{n} & \geq \sigma_{n}, \\
\alpha_{n} & =\left(\sum_{j=n+1}^{\infty} f\left(\sigma_{j}^{2}\right)^{2}\left\langle w, u_{j}\right\rangle^{2}\right)^{1 / 2},
\end{aligned}
$$

so provided the function $\Theta(\lambda)=\sqrt{\lambda} f(\lambda)$ is strictly monotonically increasing (e.g., if $f(\lambda)>0$ for $\lambda>0$ ), we obtain that (2.18) holds with $g_{1}=g_{2}=g_{3}=f \circ \Theta^{-1}$, which yields the usual rates under such source conditions; see, e.g., Corollary 1 in 24.

\section{THE NONLINEAR CASE}

In the linear case we have seen that the essential quantities for showing convergence are $\alpha_{n}, \beta_{n}, \gamma_{n}$ defined as in (2.6), (2.7). To deal with the nonlinear situation we define $\alpha_{n}, \beta_{n}(x), \gamma_{n}(x)$ as in (2.6), (2.7) with $T$ replaced by $F^{\prime}(x)$.

In view of the two formulations (2.2), (2.3) we consider two possible generalizations of the linear case, namely a variational one and one based on a projected operator equation.

\subsection{Nonlinear case via global minimizer.}

3.1.1. Well-definedness. First we show well-definedness of $x_{n}^{\delta}$ according to (1.3).

Proposition 3.1. Assume that $D \cap X_{n} \neq \emptyset$, and that $F$ is weakly sequentially closed, i.e., for all sequences $\left(x_{k}\right)_{k \in \mathbb{N}} \subseteq D \cap X_{n}$,

$$
\left(x_{k} \rightarrow x \wedge F\left(x_{k}\right) \rightarrow f\right) \Rightarrow(x \in D \wedge F(x)=f) .
$$

Then there exists a minimizer of the cost function $J_{n}: D \cap X_{n} \rightarrow \mathbb{R}, J\left(z_{n}\right)=$ $\left\|F\left(z_{n}\right)-y^{\delta}\right\|$.

Note that by membership of $x_{k}$ in the finite dimensional space $X_{n}$, continuity of $F$ and closedness of $D \cap X_{n}$ (see the assumptions of Proposition 3.3 and Theorem 3.4 below) are sufficient for the assumptions of Proposition 3.1 .

Proof. $J$ is coercive (i.e., boundedness of cost function values implies boundedness of arguments): By (1.5) we have for all $z_{n} \in D \cap X_{n}$ and some fixed $x_{n} \in D \cap X_{n}$,

$$
\begin{aligned}
\left\|F\left(z_{n}\right)-y^{\delta}\right\| & =\left\|F\left(z_{n}\right)-F\left(x_{n}\right)+F\left(x_{n}\right)-y^{\delta}\right\| \\
& \geq \frac{1}{1+\eta}\left\|F^{\prime}\left(z_{n}\right) P_{n}\left(z_{n}-x_{n}\right)\right\|-\left\|F\left(x_{n}\right)-y^{\delta}\right\|,
\end{aligned}
$$

hence by (2.8) (with $T$ replaced by $F^{\prime}\left(x_{n}\right)$ ) we get

$$
\begin{aligned}
\left\|z_{n}-x_{n}\right\| & =\left\|\left(F^{\prime}\left(x_{n}\right) P_{n}\right)^{\dagger} F^{\prime}\left(x_{n}\right) P_{n}\left(z_{n}-x_{n}\right)\right\| \\
& \leq \frac{1}{\gamma_{n}\left(x_{n}\right)}\left((1+\eta) J\left(z_{n}\right)+\left\|F\left(x_{n}\right)-y^{\delta}\right\|\right) .
\end{aligned}
$$

Moreover, $J_{n}$ is weakly lower semicontinuous due to weak sequential closedness of $F$ and weak lower semicontinuity of the norm.

By a standard argument we therefore get existence of a minimizer. 
In the rest of this subsection we assume that $x_{n}^{\delta} \in X_{n}$ is defined as a (not necessarily unique) global minimizer of (1.3) according to Proposition 3.1 and that

$$
P_{n} x^{\dagger} \in D, \quad n \in \mathbb{N} .
$$

Lemma 3.2. Let $F$ be continuous, $P_{n} x^{\dagger} \rightarrow x^{\dagger}$ as $n \rightarrow \infty$, and (3.1) hold, and let for all $n x_{n}^{\delta}$ be defined by (1.3).

Then the index $n_{*}$ according to the discrepancy principle (1.4) with $\tau>1$ is well-defined.

Proof. By minimality of $x_{n}^{\delta}$ and (3.1) we get the following estimate of the residual

$$
\left\|F\left(x_{n}^{\delta}\right)-y^{\delta}\right\| \leq\left\|F\left(P_{n} x^{\dagger}\right)-y^{\delta}\right\| \leq\left\|F\left(P_{n} x^{\dagger}\right)-F\left(x^{\dagger}\right)\right\|+\delta .
$$

Hence, by continuity of $F$ and $P_{n} x^{\dagger} \rightarrow x^{\dagger}$ as $n \rightarrow \infty$ we get $\left\|F\left(x_{n}^{\delta}\right)-y^{\delta}\right\| \leq \tau \delta$ for $n$ sufficiently large.

The following estimate of the error will be useful in our stability and convergence proofs: By (1.5) we have

$$
\begin{aligned}
& \left\|F^{\prime}\left(x_{n}^{\delta}\right) P_{n}\left(x_{n}^{\delta}-x^{\dagger}\right)\right\|-\left\|F^{\prime}\left(x_{n}^{\delta}\right)\left(I-P_{n}\right) x^{\dagger}\right\| \\
& \quad \leq\left\|F^{\prime}\left(x_{n}^{\delta}\right)\left(x_{n}^{\delta}-x^{\dagger}\right)\right\| \leq(1+\eta)\left(\left\|F\left(x_{n}^{\delta}\right)-y^{\delta}\right\|+\delta\right),
\end{aligned}
$$

hence by (2.7) with $T$ replaced by $F^{\prime}\left(x_{n}^{\delta}\right)$, (2.8), and (3.2)

$$
\begin{aligned}
\left\|x_{n}^{\delta}-x^{\dagger}\right\| \leq & \left\|x_{n}^{\delta}-P_{n} x^{\dagger}\right\|+\left\|\left(I-P_{n}\right) x^{\dagger}\right\| \\
\leq & \frac{1}{\gamma_{n}\left(x_{n}^{\delta}\right)}\left(\left\|F^{\prime}\left(x_{n}^{\delta}\right)\left(I-P_{n}\right) x^{\dagger}\right\|+(1+\eta)\left(\left\|F\left(x_{n}^{\delta}\right)-y^{\delta}\right\|+\delta\right)\right) \\
& +\left\|\left(I-P_{n}\right) x^{\dagger}\right\| .
\end{aligned}
$$

\subsubsection{Stability for fixed discretization level.}

Proposition 3.3. Let $F$ be continuous and satisfy (1.5), fix $n$, and let $\left(y^{k}\right)_{k \in \mathbb{N}}$ be a sequence converging to $y^{\delta}:\left\|y^{k}-y^{\delta}\right\| \rightarrow 0$ as $k \rightarrow \infty$, denote by $x_{n}^{k}$ the corresponding regularized solutions according to (1.3) with $y^{\delta}$ replaced by $y^{k}$ and assume that $D \cap X_{n}$ is closed and nonempty.

Then $\left(x_{n}^{k}\right)_{k \in \mathbb{N}}$ has a convergent subsequence and the limit of any convergent subsequence of $\left(x_{n}^{k}\right)_{k \in \mathbb{N}}$ is a global minimizer of (1.3). If this global minimizer is unique, then

$$
\left\|x_{n}^{k}-x_{n}^{\delta}\right\| \rightarrow 0 \quad \text { as } k \rightarrow \infty .
$$

Proof. On one hand, analogously to (3.2), we get by minimality of $x_{n}^{k}$ and $x_{n}^{\delta}$,

$$
\begin{aligned}
\left\|F\left(x_{n}^{k}\right)-y^{k}\right\| & \leq\left\|F\left(x_{n}^{\delta}\right)-y^{k}\right\| \leq\left\|F\left(x_{n}^{\delta}\right)-y^{\delta}\right\|+\left\|y^{k}-y^{\delta}\right\|, \\
\left\|F\left(x_{n}^{\delta}\right)-y^{\delta}\right\| & \leq\left\|F\left(x_{n}^{k}\right)-y^{\delta}\right\| \leq\left\|F\left(x_{n}^{k}\right)-y^{k}\right\|+\left\|y^{k}-y^{\delta}\right\|,
\end{aligned}
$$

hence

$$
\left|\left\|F\left(x_{n}^{k}\right)-y^{k}\right\|-\left\|F\left(x_{n}^{\delta}\right)-y^{\delta}\right\|\right| \leq\left\|y^{k}-y^{\delta}\right\| \rightarrow 0 \text { as } k \rightarrow \infty .
$$


On the other hand, for some fixed $x_{n} \in D \cap X_{n}$, we get by minimality,

$$
\begin{aligned}
\left\|F^{\prime}\left(x_{n}^{\delta}\right)\left(x_{n}^{k}-x_{n}^{\delta}\right)\right\| & \leq(1+\eta)\left\|F\left(x_{n}^{k}\right)-F\left(x_{n}^{\delta}\right)\right\| \\
& \leq(1+\eta)\left(\left\|F\left(x_{n}^{k}\right)-y^{k}\right\|+\left\|F\left(x_{n}^{\delta}\right)-y^{\delta}\right\|+\left\|y^{k}-y^{\delta}\right\|\right) \\
& \leq(1+\eta)\left(\left\|F\left(x_{n}\right)-y^{k}\right\|+\left\|F\left(x_{n}\right)-y^{\delta}\right\|+\left\|y^{k}-y^{\delta}\right\|\right) \\
& \leq 2(1+\eta)\left(\left\|F\left(x_{n}\right)-y^{\delta}\right\|+c\right)
\end{aligned}
$$

where $c$ is such that (by convergence) $\left\|y^{k}-y^{\delta}\right\| \leq c$, hence by (2.7) with $T$ replaced by $F^{\prime}\left(x_{n}^{\delta}\right)$ and (2.8),

$$
\left\|x_{n}^{k}-x_{n}^{\delta}\right\| \leq \frac{1+\eta}{\gamma_{n}\left(x_{n}^{\delta}\right)}\left(2\left\|F\left(x_{n}\right)-y^{\delta}\right\|+2 c\right)=: r,
$$

so the sequence $x_{n}^{k}$ lies in the bounded closed and finite dimensional, hence compact, set $\overline{B_{r}\left(x_{n}^{\delta}\right)} \cap X_{n}$ and therefore has a convergent subsequence $\left(x_{n}^{k_{m}}\right)_{m \in \mathbb{N}}$. For any convergent subsequence $\left(x_{n}^{k_{m}}\right)_{m \in \mathbb{N}}$ of $\left(x_{n}^{k}\right)_{k \in \mathbb{N}}$ with limit $\tilde{x}$, by (3.5) and a standard argument (see [3]) from $\left\|y^{k}-y^{\delta}\right\| \rightarrow 0$, continuity of $F$ and closedness of $D \cap X_{n}$ it follows that $\tilde{x}$ lies in $D \cap X_{n}$ and is a global minimizer of $\left\|F(\cdot)-y^{\delta}\right\|$ over $D \cap X_{n}$. Namely, we have by minimality of $x_{n}^{k}$,

$$
\begin{aligned}
\forall x_{n} \in D \cap X_{n}:\left\|F\left(x_{n}\right)-y^{\delta}\right\| & \geq \limsup _{k \rightarrow \infty}\left(\left\|F\left(x_{n}\right)-y^{k}\right\|-\left\|y^{k}-y^{\delta}\right\|\right) \\
& \geq \limsup _{k \rightarrow \infty}\left(\left\|F\left(x_{n}^{k}\right)-y^{k}\right\|-\left\|y^{k}-y^{\delta}\right\|\right) \\
& \geq\left\|F(\tilde{x})-y^{\delta}\right\| .
\end{aligned}
$$

In case of uniqueness of this global minimizer, a subsequence subsequence argument yields convergence.

\subsubsection{Convergence of global minimizers.}

Theorem 3.4. Let $F$ be continuous and satisfy (1.5), assume that for all $n \in \mathbb{N}$, $D \cap X_{n}$ is closed and nonempty, that (3.1) holds and that $x_{n}^{\delta}$ is defined as a solution to $(1.3)$.

If

$$
\sup _{x \in D \cap X_{n}} \frac{\beta_{n}(x)}{\gamma_{n}(x)} \rightarrow 0, \quad \sup _{\tilde{x}, x \in D \cap X_{n}} \frac{\beta_{n-1}(\tilde{x})}{\gamma_{n}(x)} \rightarrow 0, \quad \alpha_{n} \rightarrow 0, \quad \text { as } n \rightarrow \infty,
$$

then for $n_{*}=n_{*}(\delta)$ according to (1.4),

$$
\left\|x_{n_{*}(\delta)}^{\delta}-x^{\dagger}\right\| \rightarrow 0 \text { as } \delta \rightarrow 0 .
$$

Remark 3.5. Instead of (3.6) we actually need

$$
\frac{\beta_{n}\left(x_{n}^{\delta}\right)}{\gamma_{n}\left(x_{n}^{\delta}\right)} \rightarrow 0, \quad \frac{\beta_{n-1}\left(P_{n-1} x^{\dagger}\right)}{\gamma_{n}\left(x_{n}^{\delta}\right)} \rightarrow 0, \quad \alpha_{n} \rightarrow 0, \quad \text { as } n \rightarrow \infty .
$$

Proof. Analogously to the linear case we distinguish between two cases:

If $n_{m} \rightarrow \infty$ as $m \rightarrow \infty$, then we use (3.2) to obtain

$$
\begin{aligned}
\tau \delta_{m} \leq\left\|F\left(x_{n_{m}-1}^{\delta_{m}}\right)-y_{m}\right\| & \leq\left\|F\left(P_{n_{m}-1} x^{\dagger}\right)-F\left(x^{\dagger}\right)\right\|+\delta_{m} \\
& \leq \frac{1}{1-\eta}\left\|F^{\prime}\left(P_{n_{m}-1} x^{\dagger}\right)\left(P_{n_{m}-1} x^{\dagger}-x^{\dagger}\right)\right\|+\delta_{m},
\end{aligned}
$$


hence

so by (3.4) we get

$$
\frac{\delta_{m}}{\gamma_{n_{m}}\left(x_{n_{m}}^{\delta_{m}}\right)} \leq \frac{1}{\tau-1} \frac{1}{1-\eta} \frac{\beta_{n_{m}-1}\left(P_{n_{m}-1} x^{\dagger}\right)}{\gamma_{n_{m}}\left(x_{n_{m}}^{\delta_{m}}\right)}
$$

$$
\left\|x_{n_{m}}^{\delta_{m}}-x^{\dagger}\right\| \leq \frac{\beta_{n_{m}}\left(x_{n_{m}}^{\delta_{m}}\right)}{\gamma_{n_{m}}\left(x_{n_{m}}^{\delta_{m}}\right)}+\frac{1+\eta}{1-\eta} \frac{\tau+1}{\tau-1} \frac{\beta_{n_{m}-1}\left(P_{n_{m}-1} x^{\dagger}\right)}{\gamma_{n_{m}}\left(x_{n_{m}}^{\delta_{m}}\right)}+\alpha_{n_{m}} \rightarrow 0
$$

as $m \rightarrow \infty$.

If there exists an $N \in \mathbb{N}$ and a subsequence $\left(m_{k}\right)_{k \in \mathbb{N}}$ such that $n_{m_{k}}=N$ for all $k \in \mathbb{N}$, we get

$$
\tau \delta_{m_{k}} \geq\left\|F\left(x_{n_{m_{k}}}^{\delta_{m_{k}}}\right)-y_{m_{k}}\right\|=\left\|F\left(x_{N}^{\delta_{m_{k}}}\right)-y_{m_{k}}\right\|,
$$

hence taking the limit on both sides and using stability for fixed $N$ (see Proposition 3.3) we get that $x_{N}^{\delta_{m_{k}}}$ has a convergent subsequence and the limit $\tilde{x}$ of any convergent subsequence of $x_{N}^{\delta_{m_{k}}}$ satisfies $F(\tilde{x})=y$, so $\tilde{x}$ is a solution.

By uniqueness of this solution, a subsequence subsequence argument yields convergence.

\subsection{Nonlinear case via Euler equation.}

3.2.1. Well-definedness. If the global minimizer according to Proposition 3.1 lies in the interior of $D$, it will also satisfy the Euler equation

$$
\left(F^{\prime}\left(x_{n}^{\delta}\right) P_{n}\right)^{*}\left(F\left(x_{n}^{\delta}\right)-y^{\delta}\right)=0,
$$

which implies

$$
Q_{n}\left(x_{n}^{\delta}\right)\left(F\left(x_{n}^{\delta}\right)-y^{\delta}\right)=0 \quad \text { with } Q_{n}(x)=\operatorname{Proj}_{Y_{n}(x)}, Y_{n}(x):=F^{\prime}(x) X_{n} .
$$

Our following investigations on the formulation (3.8) are also motivated by the fact that a numerical optimization method will usually not yield a global minimizer but a stationary point.

In this subsection we assume that $x_{n}^{\delta}$ is defined (not necessarily uniquely) by (3.9), which is possible, e.g., under the assumptions of Proposition 3.1 provided the minimizer lies in the interior of $D$, or alternatively by a fixed point argument analogously to Lemma 2 in 12 .

Again we start with a result on the discrepancy principle.

Lemma 3.6. Let (1.5) be satisfied, $F^{\prime}(x)$ be uniformly bounded on D, let $P_{n} x^{\dagger} \rightarrow$ $x^{\dagger}$ as $n \rightarrow \infty$, and for all $n$ let $x_{n}^{\delta}$ be defined by (3.9).

Then the index $n_{*}$ according to the discrepancy principle (1.4) with $\tau>\frac{1+\eta}{1-\eta}$ is well-defined.

Proof.

$$
\begin{aligned}
F\left(x_{n}^{\delta}\right)-y^{\delta}= & \left(I-Q_{n}\left(x_{n}^{\delta}\right)\right)\left(F\left(x_{n}^{\delta}\right)-y^{\delta}\right) \\
= & \left(I-Q_{n}\left(x_{n}^{\delta}\right)\right) \cdot \\
& \left(F^{\prime}\left(x_{n}^{\delta}\right)\left(x_{n}^{\delta}-x^{\dagger}\right)+F\left(x_{n}^{\delta}\right)-F\left(x^{\dagger}\right)-F^{\prime}\left(x_{n}^{\delta}\right)\left(x_{n}^{\delta}-x^{\dagger}\right)+y-y^{\delta}\right) \\
= & \left(I-Q_{n}\left(x_{n}^{\delta}\right)\right) . \\
& \left(F^{\prime}\left(x_{n}^{\delta}\right)\left(P_{n} x^{\dagger}-x^{\dagger}\right)+F\left(x_{n}^{\delta}\right)-F\left(x^{\dagger}\right)-F^{\prime}\left(x_{n}^{\delta}\right)\left(x_{n}^{\delta}-x^{\dagger}\right)+y-y^{\delta}\right)
\end{aligned}
$$


since $\left(I-Q_{n}\left(x_{n}^{\delta}\right)\right) F^{\prime}\left(x_{n}^{\delta}\right) x_{n}^{\delta}=0=\left(I-Q_{n}\left(x_{n}^{\delta}\right)\right) F^{\prime}\left(x_{n}^{\delta}\right) P_{n} x^{\dagger}$, hence, due to (1.5)

$$
\left\|F\left(x_{n}^{\delta}\right)-y^{\delta}\right\| \leq \frac{1}{1-\eta}\left(\left\|\left(I-Q_{n}\left(x_{n}^{\delta}\right)\right) F^{\prime}\left(x_{n}^{\delta}\right)\left(I-P_{n}\right) x^{\dagger}\right\|+(1+\eta) \delta\right)
$$

and therewith $\left\|F\left(x_{n}^{\delta}\right)-y^{\delta}\right\| \leq \tau \delta$ for $n$ sufficiently large.

The error decomposition (2.9) in the nonlinear case becomes

$$
\begin{aligned}
x_{n}^{\delta}-x^{\dagger}= & \left(F^{\prime}\left(x_{n}^{\delta}\right) P_{n}\right)^{\dagger}\left(y^{\delta}-y\right)+\left(F^{\prime}\left(x_{n}^{\delta}\right) P_{n}\right)^{\dagger} F^{\prime}\left(x_{n}^{\delta}\right)\left(I-P_{n}\right) x^{\dagger}-\left(I-P_{n}\right) x^{\dagger} \\
& +\left(F^{\prime}\left(x_{n}^{\delta}\right) P_{n}\right)^{\dagger}\left(F\left(x^{\dagger}\right)-F\left(x_{n}^{\delta}\right)-F^{\prime}\left(x_{n}^{\delta}\right)\left(x^{\dagger}-x_{n}^{\delta}\right)\right),
\end{aligned}
$$

where we have used the identity

$$
\left(F^{\prime}\left(x_{n}^{\delta}\right) P_{n}\right)^{\dagger}\left(y^{\delta}-F\left(x_{n}^{\delta}\right)\right)=\left(F^{\prime}\left(x_{n}^{\delta}\right) P_{n}\right)^{\dagger} Q_{n}\left(x_{n}^{\delta}\right)\left(y^{\delta}-F\left(x_{n}^{\delta}\right)\right)=0
$$

and (2.8).

\subsubsection{Stability for fixed discretization level.}

Proposition 3.7. Let $n$ be fixed and let $\left(y^{k}\right)_{k \in \mathbb{N}}$ be a sequence converging to $y^{\delta}:\left\|y^{k}-y^{\delta}\right\| \rightarrow 0$ as $k \rightarrow \infty$ and denote by $x_{n}^{k}$ the corresponding regularized solutions according to (3.9) with $y^{\delta}$ replaced by $y^{k}$. Additionally, assume that $F$, $F^{\prime}$ are continuous, satisfy (1.5), that $D \cap X_{n}$ is closed, and that

$$
\exists \underline{\gamma}_{n}>0 \forall x \in D \cap X_{n}: \gamma_{n}(x) \geq \underline{\gamma}_{n}>0
$$

as well as

$$
\exists \bar{\beta}_{n}>0 \forall x \in D \cap X_{n}: \beta_{n}(x) \leq \bar{\beta}_{n}
$$

holds.

Then $\left(x_{n}^{k}\right)_{k \in \mathbb{N}}$ has a convergent subsequence and the limit of any convergent subsequence of $\left(x_{n}^{k}\right)_{k \in \mathbb{N}}$ solves (3.9). If this solution is unique, then

$$
\left\|x_{n}^{k}-x_{n}^{\delta}\right\| \rightarrow 0 \text { as } k \rightarrow \infty \text {. }
$$

Proof. From (3.11), (3.12), which remain valid under the present assumptions, we obtain

$$
\begin{aligned}
\left\|x_{n}^{k}-P_{n} x^{\dagger}\right\| & \leq \frac{1}{\gamma_{n}\left(x_{n}^{k}\right)}\left(\left\|y^{k}-y\right\|+\beta_{n}\left(x_{n}^{k}\right)+\eta\left\|F\left(x_{n}^{k}\right)-F\left(x^{\dagger}\right)\right\|\right) \\
& \leq \frac{1}{\gamma_{n}\left(x_{n}^{k}\right)}\left(\delta_{k}+\beta_{n}\left(x_{n}^{k}\right)+\eta\left(\delta_{k}+\frac{1}{1-\eta}\left(\beta_{n}\left(x_{n}^{k}\right)+(1+\eta) \delta_{k}\right)\right)\right)
\end{aligned}
$$

with $\delta_{k}:=\left\|y^{k}-y^{\delta}\right\|+\delta$, which by (3.13), (3.14) implies boundedness of $x_{n}^{k}$. Hence by compactness of balls in the finite dimensional subspace $X_{n}$ we get existence of a convergent subsequence of $x_{n}^{k}$. By continuity of $F$ and $F^{\prime}$, as well as $\left(F^{\prime}\left(x_{n}^{k}\right) P_{n}\right)^{*}\left(F\left(x_{n}^{k}\right)-y_{n}^{k}\right)=0$, the limit $\tilde{x}$ of any convergent subsequence of $x_{n}^{k}$ satisfies $\left(F^{\prime}(\tilde{x}) P_{n}\right)^{*}\left(F(\tilde{x})-y^{\delta}\right)=0$. 


\subsubsection{Convergence of solutions to Euler equations.}

Theorem 3.8. Assume that $x_{n}^{\delta}$ is defined as a solution to (3.9), that $F, F^{\prime}$ are continuous with $F^{\prime}(x)$ uniformly bounded and satisfy (1.5), that $D \cap X_{n}$ is closed, and that

$$
\sup _{x \in D \cap X_{n}} \frac{\beta_{n}(x)}{\gamma_{n}(x)} \rightarrow 0, \quad \sup _{\tilde{x}, x \in D \cap X_{n}} \frac{\beta_{n-1}(\tilde{x})}{\gamma_{n}(x)} \rightarrow 0, \quad \alpha_{n} \rightarrow 0, \quad \text { as } n \rightarrow \infty
$$

holds.

Then for $n_{*}=n_{*}(\delta)$ according to (1.4),

$$
\left\|x_{n_{*}(\delta)}^{\delta}-x^{\dagger}\right\| \rightarrow 0 \text { as } \delta \rightarrow 0 .
$$

Remark 3.9. More precisely, we actually need

$$
\frac{\beta_{n}\left(x_{n}^{\delta}\right)}{\gamma_{n}\left(x_{n}^{\delta}\right)} \rightarrow 0, \quad \frac{\beta_{n-1}\left(x_{n-1}^{\delta}\right)}{\gamma_{n}\left(x_{n}^{\delta}\right)} \rightarrow 0, \quad \alpha_{n} \rightarrow 0, \quad \text { as } n \rightarrow \infty
$$

in place of (3.15).

Proof. With (3.12), by (1.5) we get

$$
\begin{aligned}
\left\|x_{n}^{\delta}-x^{\dagger}\right\| & \leq \frac{\delta}{\gamma_{n}\left(x_{n}^{\delta}\right)}+\frac{\beta_{n}\left(x_{n}^{\delta}\right)}{\gamma_{n}\left(x_{n}^{\delta}\right)}+\alpha_{n}+\frac{\eta\left\|F\left(x_{n}^{\delta}\right)-F\left(x^{\dagger}\right)\right\|}{\gamma_{n}\left(x_{n}^{\delta}\right)} \\
& \leq \frac{1}{1-\eta} \frac{\delta}{\gamma_{n}\left(x_{n}^{\delta}\right)}+\frac{1}{1-\eta} \frac{\beta_{n}\left(x_{n}^{\delta}\right)}{\gamma_{n}\left(x_{n}^{\delta}\right)}+\alpha_{n}
\end{aligned}
$$

where analogously to (3.10), 3.11) we have

$$
\begin{aligned}
F\left(x_{n}^{\delta}\right)-F\left(x^{\dagger}\right)=\left(I-Q_{n}\left(x_{n}^{\delta}\right)\right)( & F^{\prime}\left(x_{n}^{\delta}\right)\left(P_{n} x^{\dagger}-x^{\dagger}\right)+F\left(x_{n}^{\delta}\right) \\
& \left.-F\left(x^{\dagger}\right)-F^{\prime}\left(x_{n}^{\delta}\right)\left(x_{n}-x^{\dagger}\right)\right)+Q_{n}\left(y^{\delta}-y\right),
\end{aligned}
$$

hence, using once more (1.5),

$$
\left\|F\left(x_{n}^{\delta}\right)-F\left(x^{\dagger}\right)\right\| \leq \frac{1}{1-\eta}\left(\left\|\left(I-Q_{n}\left(x_{n}^{\delta}\right)\right) F^{\prime}\left(x_{n}^{\delta}\right)\left(P_{n} x^{\dagger}-x^{\dagger}\right)\right\|+\delta\right) .
$$

The rest of the proof goes analogously to the linear case:

In case of $n_{m} \rightarrow \infty$, in place of (2.12), we get by (3.11),

$$
\tau \delta_{m} \leq\left\|F\left(x_{n_{m}-1}^{\delta_{m}}\right)-y_{m}\right\| \leq \frac{1}{1-\eta}\left(\|\left(F^{\prime}\left(x_{n_{m}-1}^{\delta_{m}}\right)\left(I-P_{n_{m}-1}\right) x^{\dagger} \|+(1+\eta) \delta_{m}\right),\right.
$$

and therewith, instead of (2.13),

$$
\frac{\delta_{m}}{\gamma_{n_{m}}\left(x_{n_{m}}^{\delta_{m}}\right)} \leq \frac{1}{\tau-\frac{1+\eta}{1-\eta}} \frac{\beta_{n_{m}-1}\left(x_{n_{m}-1}^{\delta_{m}}\right)}{\gamma_{n_{m}}\left(x_{n_{m}}^{\delta_{m}}\right)} .
$$

For the case with a finite accumulation point $N$ of the discretization levels $n_{m}$, we estimate, using (3.10),

$\tau \delta_{m_{k}} \geq\left\|F\left(x_{n_{m_{k}}}^{\delta_{m_{k}}}\right)-y_{m_{k}}\right\| \geq \frac{1}{1+\eta}\left\|\left(I-Q_{N}\left(x_{N}^{\delta_{m_{k}}}\right)\right) F^{\prime}\left(x_{N}^{\delta_{m_{k}}}\right)\left(I-P_{N}\right) x^{\dagger}\right\|-(1+\eta) \delta_{m_{k}}$,

hence

$$
(\tau+\eta+1)(1+\eta) \delta_{m_{k}} \geq\left\|\left(I-Q_{N}\left(x_{N}^{\delta_{m_{k}}}\right)\right) F^{\prime}\left(x_{N}^{\delta_{m_{k}}}\right)\left(I-P_{N}\right) x^{\dagger}\right\|
$$


Taking on both sides the limit along a subsequence $k_{l}$ for which $x_{N}^{\delta_{m_{k}}}$ converges to some $x_{N}^{0}$ according to Proposition 3.7 and arguing similar to the stability proof, we get

$$
0=\left\|\left(I-Q_{N}\left(x_{N}^{0}\right)\right) F^{\prime}\left(x_{N}^{0}\right)\left(I-P_{N}\right) x^{\dagger}\right\|,
$$

which analogously to the linear case implies $\left(I-P_{N}\right) x^{\dagger}=0$, hence convergence.

Remark 3.10. By inspection of the proof we see that instead of solving (3.8) exactly, it suffices to find $x_{n}^{\delta}$ such that

$$
\left\|\left(F^{\prime}\left(x_{n}^{\delta}\right) P_{n}\right)^{*}\left(F\left(x_{n}^{\delta}\right)-y^{\delta}\right)\right\| \leq c \gamma_{n}\left(x_{n}^{\delta}\right) \delta,
$$

with $0<c<\tau(1-\eta)-(1+\eta)$. In our computations we use the heuristic tolerance

$$
\left\|\left(F^{\prime}\left(x_{n}^{\delta}\right) P_{n}\right)^{*}\left(F\left(x_{n}^{\delta}\right)-y^{\delta}\right)\right\| \leq c \delta^{2},
$$

which cannot be shown to yield convergence, since in place of (3.18) we get an expression that is quadratic in $\frac{\delta}{\gamma_{n}\left(x_{n}^{\delta}\right)}$, namely

$$
\frac{\delta_{m}}{\gamma_{n_{m}}\left(x_{n_{m}}^{\delta_{m}}\right)} \leq \frac{1}{\tau-\frac{1+\eta}{1-\eta}} \frac{\beta_{n_{m}-1}\left(x_{n_{m}-1}^{\delta_{m}}\right)}{\gamma_{n_{m}}\left(x_{n_{m}}^{\delta_{m}}\right)}+c\left(\frac{\delta_{m}}{\gamma_{n_{m}}\left(x_{n_{m}}^{\delta_{m}}\right)}\right)^{2},
$$

which does not exclude large values of $\frac{\delta}{\gamma_{n}\left(x_{n}^{\delta}\right)}$.

Condition (3.19) (or (3.20) can be used as a stopping criterion in the iterative solution of the Euler equation.

\section{NumericAl RESUlts}

4.1. Test problem. For illustrating the theoretical results from above, we consider the test problem of identifying the source term $q$ in the PDE

$$
\begin{aligned}
& -\Delta u+q u=f \quad \text { in } \Omega, \\
& u=0 \quad \text { on } \partial \Omega
\end{aligned}
$$

on the unit square $\Omega=[0,1] \times[0,1] \subset \mathbb{R}^{2}$ from measurements $u$ in the whole domain $\Omega$. Defining $F$ as the parameter-to-solution map for the PDE (4.1) leads to a nonlinear inverse problem

$$
F(q)=u
$$

where $q \in X=L^{2}(\Omega), u \in Y=L^{2}(\Omega)$, and $F: X \rightarrow Y$, whose properties have been studied, e.g., in 3], 9], where also the Scherzer condition (1.5) has been verified. This problem has been used as a model problem for testing numerical methods for nonlinear inverse problems in many papers. In order to achieve a nonempty interior of the domain, similarly to [9], we set

$$
D=\left\{q \in L^{2}(\Omega) \mid\|q-\hat{q}\|_{L^{2}(\Omega)} \leq \beta \text { for some } \hat{q} \in L^{\infty}(\Omega) \text { with } \hat{q} \geq 0 \text { a.e. }\right\}
$$

where $\frac{1}{\beta}$ is larger than the norm of the continuous embedding $H_{0}^{1}(\Omega) \rightarrow L^{4}(\Omega)$.

Obviously, the range of the forward operator $F$, mapping a parameter $q$ to the exact solution of the PDE problem (4.1) is actually $H^{2}(\Omega) \cap H_{0}^{1}(\Omega)$ due to convexity of $\Omega$ (cf., e.g., [5]). On the other hand, only values but not derivatives of $u$ can be measured, so the natural choice of $Y$ as a Hilbert space is $L^{2}(\Omega)$. This discrepancy of smoothness yields an ill-posedness of degree two of the inverse problem, which can also be seen directly from the fact that we consider $q$ and $u$ in the same space, but $q$ is determined from $u$ by application of the Laplace operator. 
We consider three test cases for the parameter $q$, which in the first two cases is defined as a Gaussian density function:

$$
q^{\dagger}(x, y)=\frac{1}{2 \pi\|\Sigma\|} e^{\frac{(x-\mu)^{T} \Sigma^{-1}(y-\mu)}{2}}
$$

with $\Sigma=0.005 I d$, and $\Sigma=0.03 I d$, respectively, where in both cases $\mu=\left(\frac{1}{2}, \frac{1}{2}\right)$. The third test parameter is piecewise constant and supported on three nonintersecting circles

$$
q^{\dagger}(x, y)= \begin{cases}2 & \text { if }(x, y) \in K_{1} \cup K_{2} \cup K_{3}, \\ 0 & \text { else, }\end{cases}
$$

where $K_{1}, K_{2}, K_{3}$ are three circles with radius $r=0.05$ and midpoints $M_{1}\left(\frac{1}{4} / \frac{1}{4}\right)$, $M_{2}\left(\frac{1}{4} / \frac{3}{4}\right)$ and $M_{3}\left(\frac{3}{4} / \frac{3}{4}\right)$. See Figure 1 for a plot of the three test parameters on a very fine grid, with about 16.000 triangles. The corresponding solutions $u$ of (4.1) are shown in Figure 2
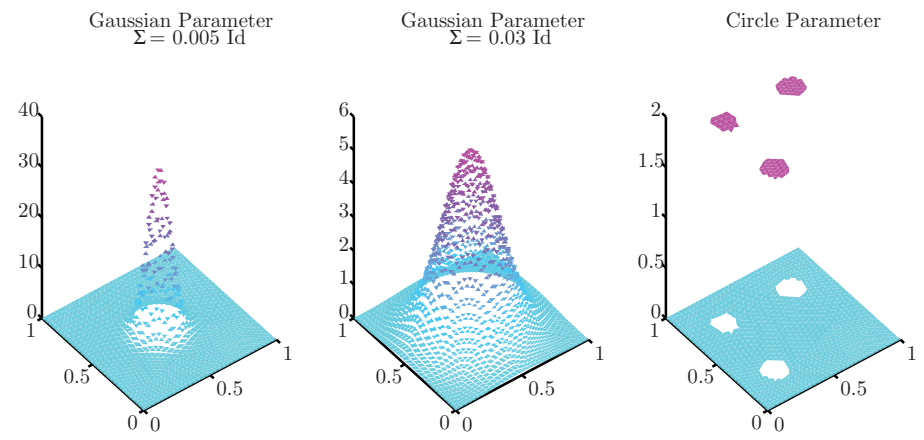

FiguRE 1. Exact parameter on fine grid.

$$
\begin{gathered}
\text { Gaussian Parameter } \\
\Sigma=0.005 \mathrm{ld}
\end{gathered}
$$

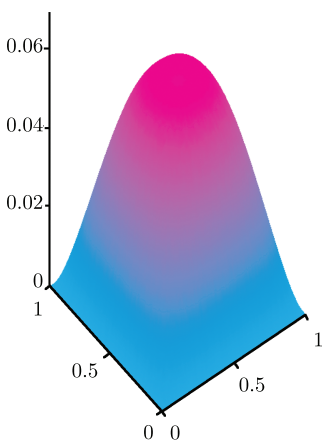

Gaussian Parameter $\Sigma=0.03$ ld

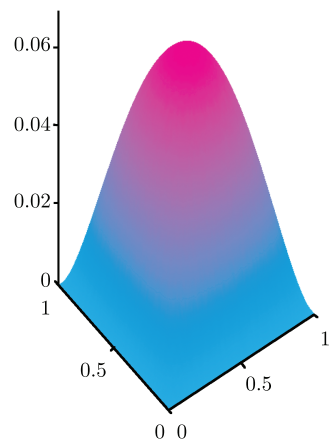

Circle Parameter

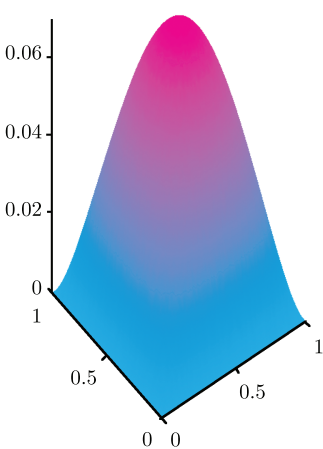

FIGURE 2. Exact solutions to the corresponding source parameter on fine grid. 
4.2. Numerical Implementation. For generating synthetic data and for solving the forward problem (4.1) we use the Finite Element Method as provided in a Matlab routine, that is capable of generating different meshes $\left(p_{n}, t_{n}\right)$, consisting of the set of points $p_{n}$ and the set of triangles $t_{n}$. Since the discretizations of the parameter $q$ are defined on the triangles and those of the PDE solutions on the set of points, the natural choice of $X_{n}$ is the space of piecewise constant functions with possible jumps over the triangle edges, whereas by choosing a very fine mesh for $u$ we get - up to a small approximation error - an exact PDE solution. Considering $L$ levels of discretization, labeling by 1 the coarsest and by $L$ the finest one, we carry out the PDE solution for data generation on level $L$, whereas all other PDE solutions are computed on level $L-1$ in order to avoid an inverse crime (see e.g., [11, 21]). For the projection spaces $X_{n}$ the level $n$ runs from 1 to $L-1$.

Hence the discretized forward operator consists of two components: interpolation from mesh $\left(p_{n}, t_{n}\right)$ to mesh $\left(p_{L-1}, t_{L-1}\right)$ and solving the underlying boundary value problem on the finest mesh.

In order to be able to transfer information on the parameter $q$ over different grid levels in an exact manner, we generate nested grids, i.e., such that

$$
p_{n-1} \subseteq p_{n} \text { and } \forall \omega \in t_{n} \exists \tilde{\omega} \in t_{n-1}: \omega \subseteq \tilde{\omega}, \quad \text { for } n=2, \ldots, L-1
$$

where $\omega, \tilde{\omega}$ denote triangles. Again, in order to avoid an inverse crime, the finest mesh for data generation is designed such that it is not part of the hierarchical mesh structure. For this purpose we initialize our computations by generating a hierarchical sequence of grids and one additional very fine grid for data generation, as well as by computing a PDE solution on the finest grid, which is then corrupted with random noise of a given percentage of the data norm as listed in the tables below. Then we solve (4.2) with $q_{n}$ element of different subspaces $X_{n}$ (given by respective meshes) as described in the previous section. Summarizing, we have implemented the following procedure:

(1) Generate $L$ finite element meshes $\left(p_{1}, t_{1}\right), \ldots,\left(p_{L}, t_{L}\right)$ satisfying (4.4).

(2) Solve (4.1) with given $q^{\dagger}$ on mesh $L$ to get data $u$.

(3) Interpolate $u$ to the $L-1$-th mesh to avoid an inverse crime.

(4) Add random noise to $u$ according to the desired noise level $\delta$ in order to get $u^{\delta}$.

(5) Solve (1.3) with $X_{n}$ defined by $t_{n}, n=1, \ldots, L-1$.

For validating the regularizing property of discretization in preimage space, we solve (4.2) in a least-squares sense, i.e., such that $\left\|F(q)-u^{\delta}\right\|^{2}$ is minimal, which is the method described and analyzed in the previous sections of this paper. For the minimization of the least squares functional we used a Quasi-Newton algorithm with BFGS update, as implemented in the Matlab function fminunc, where we provided the gradient $\left(F^{\prime}\left(x_{n}^{\delta}\right) P_{n}\right)^{*}\left(F\left(x_{n}^{\delta}\right)-y^{\delta}\right)$, which is computed using the adjoint technique. The algorithm stops if

$$
\|\nabla J(x)\|=\left\|\left(F^{\prime}\left(x_{n}^{\delta}\right) P_{n}\right)^{*}\left(F\left(x_{n}^{\delta}-y^{\delta}\right)\right)\right\| \leq c \delta^{2}
$$

according to equation (3.20) with $c=8 \cdot 10^{-3}$. 

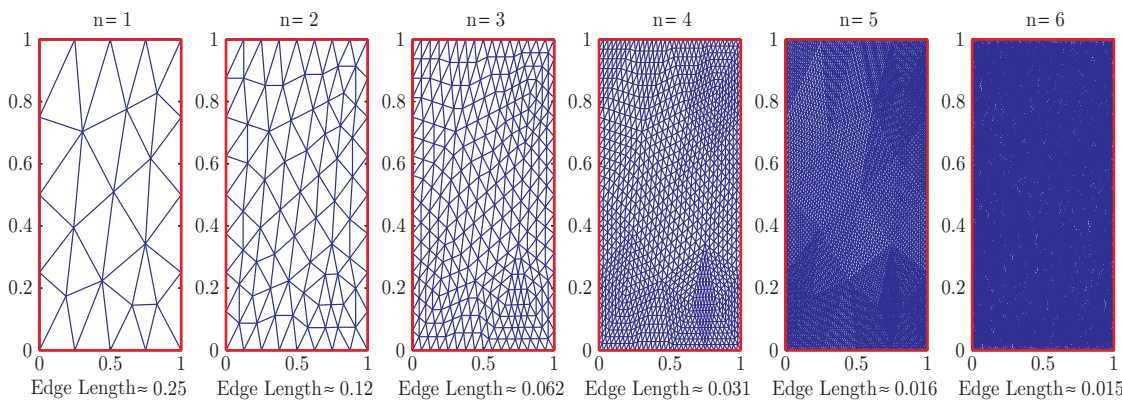

FiguRE 3 . The $L=6$ different meshes for the Gaussian parameter together with their corresponding maximum edge length.

4.3. Results. For each of the test examples, we generated a sequence of $L=6$ meshes (see Figure 3) and interpolated the data to the fifth mesh, with $\approx 4000$ triangles for the circle parameter and $\approx 9000$ triangles in case of gaussian parameters. We corrupted the exact data with white Gaussian noise according to different noise levels $\delta=0.5 \%, 1 \%, 2 \%, 4 \%, 8 \%$. Then we solved the inverse problem on each subspace $X_{n}, n=1, \ldots, L$ in the least squares sense (1.3). For comparison of the different solutions we computed the relative error:

$$
e_{r}=\frac{\left\|q-q^{\dagger}\right\|}{\left\|q^{\dagger}\right\|}
$$

with $q^{\dagger}$ given on $\left(p_{5}, t_{5}\right)$. For computing this error, we interpolated $q_{k}, k=1, \ldots, 4$ to mesh 5, such that every small triangle from the fine mesh obtains the value from its surrounding triangle of the coarser mesh. This is possible because of the hierarchical structure of the mesh sequence.

In addition, for every solution we computed the corresponding ratio between discrepancy and noise level, to see if we can numerically validate the theoretically investigated discrepancy principle:

$$
\tilde{\tau}=\frac{\left\|F(q)-u^{\delta}\right\|}{\delta} .
$$

In Table 1 we list the relative error $e_{r}$ and the associated ratio $\tilde{\tau}$ for the five different meshes and the different noise levels for the Gaussian parameter with the least squares solution. Additionally, we mention the computation time $t$ for the minimization process on the domain and the number of iterations the Quasi Newton solver carried out. There are three different features to mention.

First, if we look at a fixed noise level, e.g., $\delta=1 \%$, we see the well-known error behavior of regularization methods for ill-posed problems (see [4]): For finer grids the error gets smaller but when the mesh size gets too small the error grows again. Thus there is an optimal discretization level, for which the error is minimal.

Second, it can be seen, that we are able to validate the discrepancy principle also numerically. If we look at the smallest error (the bold numbers) we see that the corresponding ratio $\tilde{\tau} \approx 1$, which confirms our convergence analysis. Also for smaller noise level the best $n$ determining the projection space $X_{n}$ gets larger, e.g., for the Gaussian parameter with $\Sigma=0.03 I d$ and $0.5 \%$ noise, we achieved the smallest error on the finest mesh. 
Additionally, we find that for $\delta \rightarrow 0$ the estimated parameter $q$ tends towards the exact parameter $q^{\dagger}$; see the bold error numbers e.g. in Table 2

In case of the circle parameter (see Table 3), errors are higher than in the Gaussian case, even in the case of $\delta=0.25 \%$ noise, which is probably due to the nonsmoothness of the parameter.

TABle 1. Gaussian Parameter with $\Sigma=0.005 I d$

\begin{tabular}{ll||ccccc} 
Mesh & & 1 & 2 & 3 & 4 & 5 \\
Edge Length & & 0.25 & 0.125 & 0.0625 & 0.03125 & 0.016 \\
\hline \hline Noise Level & & & & & & \\
\hline $8 \%$ & $e_{r}$ & $\mathbf{0 . 8 5 7 0}$ & 0.7935 & 0.9413 & 0.9999 & 0.9999 \\
& $\tilde{\tau}$ & 1.0721 & 1.0742 & 1.8609 & 2.1985 & 2.1985 \\
& $t$ & 1.3220 & 1.3203 & 1.2083 & 0.1461 & 0.1659 \\
& $\# \mathrm{It}$ & 3 & 3 & 1 & 0 & 0 \\
\hline $4 \%$ & $e_{r}$ & 0.9533 & $\mathbf{0 . 7 8 0 9}$ & 0.8143 & 0.8778 & 0.9999 \\
& $\tilde{\tau}$ & 1.0783 & 1.1862 & 1.5464 & 2.4434 & 4.0388 \\
& $t$ & 2.8897 & 1.4680 & 1.3593 & 1.4594 & 0.1679 \\
& $\# \mathrm{It}$ & 15 & 4 & 2 & 1 & 0 \\
\hline $2 \%$ & $e_{r}$ & 0.9568 & $\mathbf{0 . 5 2 9 7}$ & 0.7710 & 0.8014 & 0.9659 \\
& $\tilde{\tau}$ & 1.2571 & 1.0665 & 1.5834 & 2.1592 & 6.9880 \\
& $t$ & 3.0220 & 2.7704 & 1.6703 & 1.9957 & 2.5002 \\
& $\# \mathrm{It}$ & 16 & 14 & 4 & 2 & 1 \\
\hline $1 \%$ & $e_{r}$ & 1.1083 & 0.5224 & $\mathbf{0 . 4 7 2 1}$ & 0.7759 & 0.8174 \\
& $\tilde{\tau}$ & 1.7815 & 1.2184 & 1.2824 & 2.8203 & 5.1237 \\
& $t$ & 6.7834 & 3.0331 & 3.0807 & 2.4399 & 8.3380 \\
& $\# \mathrm{It}$ & 45 & 16 & 13 & 3 & 2 \\
\hline $0.5 \%$ & $e_{r}$ & 1.2973 & 0.4612 & 0.4492 & $\mathbf{0 . 4 4 7 5}$ & 0.7684 \\
& $\tilde{\tau}$ & 3.0110 & 1.1056 & 1.6886 & 1.8145 & 5.2024 \\
& $t$ & 11.4500 & 6.6803 & 3.4147 & 7.1335 & 18.6847 \\
& $\# \mathrm{It}$ & 80 & 43 & 15 & 13 & 4 \\
\hline
\end{tabular}


TABle 2. Gaussian Parameter with $\Sigma=0.03 I d$

\begin{tabular}{ll||ccccc} 
Mesh & & 1 & 2 & 3 & 4 & 5 \\
Edge Length & & 0.25 & 0.125 & 0.0625 & 0.03125 & 0.016 \\
\hline \hline Noise Level & & & & & & \\
\hline $8.000 \%$ & $e_{r}$ & 0.3072 & $\mathbf{0 . 2 8 2 3}$ & 0.9994 & 0.9994 & 0.9994 \\
& $\tilde{\tau}$ & 1.0006 & 1.0241 & 1.5076 & 1.5076 & 1.5076 \\
& $t$ & 1.3217 & 1.1925 & 0.1383 & 0.1454 & 0.1669 \\
& $\# \mathrm{It}$ & 3 & 2 & 0 & 0 & 0 \\
\hline $4.000 \%$ & $e_{r}$ & 0.3012 & $\mathbf{0 . 2 1 1 9}$ & 0.2497 & 0.9994 & 0.9994 \\
& $\tilde{\tau}$ & 1.0050 & 1.0083 & 1.0797 & 2.4754 & 2.4754 \\
& $t$ & 1.3407 & 1.3273 & 1.3917 & 0.1467 & 0.1655 \\
& $\# \mathrm{It}$ & 3 & 3 & 2 & 0 & 0 \\
\hline $2.000 \%$ & $e_{r}$ & 0.3088 & 0.2159 & $\mathbf{0 . 1 8 9 6}$ & 0.2176 & 0.9994 \\
& $\tilde{\tau}$ & 1.0053 & 1.0073 & 1.0211 & 1.1550 & 4.6261 \\
& $t$ & 1.4493 & 1.4552 & 1.5153 & 1.9793 & 0.1683 \\
& $\# \mathrm{It}$ & 4 & 4 & 3 & 2 & 0 \\
\hline $1.000 \%$ & $e_{r}$ & 0.3017 & 0.2181 & 0.1900 & $\mathbf{0 . 1 8 4 8}$ & 0.2607 \\
& $\tilde{\tau}$ & 1.0022 & 1.0170 & 1.0195 & 1.0358 & 2.0177 \\
& $t$ & 1.9879 & 1.4568 & 1.6819 & 2.4417 & 8.3386 \\
& $\# \mathrm{It}$ & 8 & 4 & 4 & 3 & 2 \\
\hline $0.500 \%$ & $e_{r}$ & 0.2862 & 0.2034 & 0.1887 & 0.1838 & $\mathbf{0 . 1 8 1 6}$ \\
& $\tilde{\tau}$ & 1.0142 & 1.0259 & 1.1019 & 1.1643 & 1.3474 \\
& $t$ & 4.6533 & 2.1385 & 1.6900 & 2.4584 & 13.4880 \\
& $\# \mathrm{It}$ & 28 & 9 & 4 & 3 & 3 \\
\hline
\end{tabular}


TABle 3. Circle Parameter

\begin{tabular}{|c|c|c|c|c|c|c|}
\hline $\begin{array}{l}\text { Mesh } \\
\text { Edge Length }\end{array}$ & & $\begin{array}{c}1 \\
0.500\end{array}$ & $\begin{array}{c}2 \\
0.250\end{array}$ & $\begin{array}{c}3 \\
0.125\end{array}$ & $\begin{array}{c}4 \\
0.062\end{array}$ & $\begin{array}{c}5 \\
0.031\end{array}$ \\
\hline \multicolumn{7}{|l|}{ Noise Level } \\
\hline \multirow[t]{4}{*}{$8.000 \%$} & $e_{r}$ & 1.0165 & 0.7526 & 0.9180 & 0.8370 & 0.9519 \\
\hline & $\tilde{\tau}$ & 1.0123 & 1.0081 & 1.0745 & 1.0292 & 1.0953 \\
\hline & $t$ & 0.4930 & 0.4989 & 0.5019 & 0.6032 & 0.8584 \\
\hline & \#It & 2 & 2 & 1 & 1 & 1 \\
\hline \multirow[t]{4}{*}{$4.000 \%$} & $e_{r}$ & 1.0182 & 0.7734 & 0.9251 & 0.8501 & 0.9561 \\
\hline & $\tilde{\tau}$ & 1.0287 & 1.0190 & 1.2275 & 1.0863 & 1.2891 \\
\hline & $t$ & 0.5498 & 0.4992 & 0.5027 & 0.6034 & 0.8595 \\
\hline & \#It & 3 & 2 & 1 & 1 & 1 \\
\hline \multirow[t]{4}{*}{$2.000 \%$} & $e_{r}$ & 1.3231 & 0.6318 & 0.7663 & 0.8462 & 0.9553 \\
\hline & $\tilde{\tau}$ & 1.0854 & 1.0312 & 1.1211 & 1.3619 & 1.9426 \\
\hline & $t$ & 1.0880 & 0.7182 & 0.5602 & 0.6040 & 0.8599 \\
\hline & \#It & 13 & 6 & 2 & 1 & 1 \\
\hline \multirow[t]{4}{*}{$1.000 \%$} & $e_{r}$ & 1.2857 & 0.5813 & 0.6211 & 0.7685 & 0.7739 \\
\hline & $\tilde{\tau}$ & 1.2855 & 1.0110 & 1.1025 & 1.3473 & 1.3788 \\
\hline & $t$ & 1.1296 & 1.0419 & 0.8014 & 0.7344 & 2.0487 \\
\hline & $\# \mathrm{It}$ & 14 & 12 & 6 & 2 & 2 \\
\hline \multirow[t]{4}{*}{$0.500 \%$} & $e_{r}$ & 1.2617 & 0.5591 & 0.5157 & 0.6126 & 0.7751 \\
\hline & $\tilde{\tau}$ & 1.9447 & 1.0184 & 1.0481 & 1.3632 & 2.1241 \\
\hline & $t$ & 1.2188 & 1.4464 & 1.1634 & 1.2503 & 2.0698 \\
\hline & \#It & 15 & 19 & 12 & 6 & 2 \\
\hline \multirow[t]{4}{*}{$0.250 \%$} & $e_{r}$ & 1.2706 & 0.5562 & 0.4845 & 0.5097 & 0.6230 \\
\hline & $\tilde{\tau}$ & 3.3979 & 1.0105 & 1.0822 & 1.1901 & 2.1400 \\
\hline & $t$ & 1.9215 & 2.9264 & 1.5109 & 1.9494 & 6.3133 \\
\hline & \#It & 28 & 46 & 18 & 12 & 6 \\
\hline
\end{tabular}

\section{CONClusion AND REMARKS}

Motivated by the fact that in practice many ill-posed problems are regularized by discretization in preimage space and aware of the fact that this does not yield convergence in general, we give here some new results on conditional convergence and convergence rates for this approach, including noisy data and nonlinear problems. Future research will be devoted to an investigation of different strategies for choosing the regularization parameter and on a posteriori selecting the sequence of spaces $X_{n}$. One of them might be the refinement and coarsening algorithm proposed in [2], [18. Moreover, we will investigate multilevel and multigrid approaches in the context of regularization by discretization in preimage space; see, e.g., [13, 19, 14, 15, 16] for the case of regularization by discretization in image space. 


\section{ACKNOWLEDGEMENTS}

The authors would like to thank the German Science Foundation (DFG) for financial support of the project within the Cluster of Excellence in Simulation Technology (EXC 310/1) at the University of Stuttgart.

Moreover, the authors thank the referees for their fruitful comments, leading to an improved version of the paper.

\section{REFERENCES}

1. A.B. Bakushinsky and M. Yu Kokurin, Iterative methods for approximate solution of inverse problems, Springer, Dordrecht, 2004. MR2133802 (2006e:47025)

2. H. Ben Ameur, G. Chavent, and J. Jaffré, Refinement and coarsening indicators for adaptive parametrization: application to the estimation of hydraulic transmissivities, Inverse Problems 18 (2002), 775-794. MR 1910202 (2003c:76118)

3. Heinz W. Engl, Karl Kunisch, and Andreas Neubauer, Convergence rates for Tikhonov regularisation of nonlinear ill-posed problems, Inverse Problems 5 (1989), no. 4, 523-540. MR.1009037 (91k:65102)

4. H.W. Engl, M. Hanke, and A. Neubauer, Regularization of inverse problems, Kluwer, Dordrecht, 1996. MR1408680 (97k:65145)

5. P. Grisvard, Elliptic problems in nonsmooth domains, Pitman, 1985. MR775683 (86m:35044)

6. C.W. Groetsch, Inverse problems in mathematical sciences, Vieweg, Braunschweig, 1993. MR1247696 (94m:00008)

7. C.W. Groetsch and A. Neubauer, Convergence of a general projection method for an operator equation of the first kind, Houston Journal of Mathematics 14 (1988), 201-208. MR978726 (90b:65109)

8. U. Hämarik, E. Avi, and A. Ganina, On the solution of ill-posed problems by projection methods with a posteriori choice of the discretization level, Mathematical Modelling and Analysis 7 (2002), 241-252. MR 1952913 (2003k:65057)

9. M. Hanke, A. Neubauer, and O. Scherzer, A convergence analysis of the Landweber iteration for nonlinear ill-posed problems, Numer. Math. 72 (1995), 21-37. MR.1359706 (96i:65046)

10. B. Hofmann, P. Mathé, and S.V. Pereverzev, Regularization by projection: Approximation theoretic aspects and distance functions, J. Inverse Ill-Posed Problems 15 (2007), 527-545. MR2367863 (2008m:65155)

11. J. Kaipio and E. Somersalo, Statistical and computational inverse problems, Applied Mathematical Sciences, vol. 160, Springer, 2005. MR2102218 (2005g:65001)

12. B. Kaltenbacher, Regularization by projection with a posteriori discretization level choice for linear and nonlinear ill-posed problems, Inverse Problems 16 (2000), no. 5, 1523-1539. MR.1800607 (2001h:65070)

13. On the regularizing properties of a full multigrid method for ill-posed problems, Inverse Problems 17 (2001), 767-788. MR1861481 (2002h:65094)

14. _ $\quad$ - -cycle convergence of some multigrid methods for ill-posed problems, Mathematics of Computation 72 (2003), 1711-1730. MR1986801 (2004d:65069)

15. _ Towards global convergence for strongly nonlinear ill-posed problems via a regularizing multilevel approach, Numerical Functional Analysis and Optimization 27 (2006), 637-665. MR2246581 (2007c:65047)

16. Convergence rates of a multilevel method for the regularization of nonlinear illposed problems, Journal of Integral Equations and Applications 20 (2008), no. 2, 201-228. MR2418067 (2009e:47112)

17. B. Kaltenbacher, A. Neubauer, and O. Scherzer, Iterative regularization methods for nonlinear problems, de Gruyer, Berlin, New York, 2008, Radon Series on Computational and Applied Mathematics.

18. B. Kaltenbacher and J. Offtermatt, A refinement and coarsening indicator algorithm for finding sparse solutions of inverse problems, Inverse Problems and Imaging (2011), 391-406.

19. B. Kaltenbacher and J. Schicho, A multi-grid method with a priori and a posteriori level choice for the regularization of nonlinear ill-posed problems, Numerische Mathematik 93 (2002), 77107, DOI 10.1007/s002110100375. MR1938323(2003h:65076) 
20. A. Kirsch, An introduction to the mathematical theory of inverse problems, Springer, New York, 1996. MR1479408 (99c:34023)

21. R. Kress, Linear integral equations, Springer, Heidelberg, 1989, 2nd ed. 1999. MR1723850 (2000h:45001)

22. A.K. Louis, Inverse und schlecht gestellte probleme, Teubner, Stuttgart, 1989. MR.1002946 (90g:65075)

23. G.R. Luecke and K.R. Hickey, Convergence of approximate solutions of an operator equation, Houston Journal of Mathematics 11 (1985), no. 3, 345-354. MR808651 (87a:47019)

24. P. Mathé and N. Schöne, Regularization by projection in variable Hilbert scales, Applicable Analysis 87 (2008), 201-219. MR2394505 (2009h:47021)

25. V.A. Morozov, Regularization methods for ill-posed problems, CRC Press, Boca Raton, 1993. MR 1244325 (94g:65002)

26. F. Natterer, Regularisierung schecht gestellter Probleme durch Projektionsverfahren, Numerische Mathematik 28 (1977), 329-341. MR0488721(58:8238)

27. $(88 \mathrm{~m}: 44008)$

28. S.V. Pereverzev and S. Prössdorf, On the characterization of self-regularization properties of a fully discrete projection method for Symm's integral equation, Journal of Integral Equations and Applications 12 (2000), no. 2, 113-130. MR.1771514 (2001j:65198)

29. O. Scherzer, Convergence criteria of iterative methods based on Landweber iteration for solving nonlinear problems, Journal of Mathematical Analysis and Applications 194 (1995), no. 3, 911-933. MR 1350202 (97d:65033)

30. T.I. Seidman, Nonconvergence results for the application of least squares estimation to ill-posed problems, Journal of Optimization Theory and Applications 30 (1980), 535-547. MR.572154 (81g:65073)

31. A. N. Tikhonov and V. A. Arsenin, Methods for solving ill-posed problems, Nauka, Moscow, 1979.

32. G. Vainikko and U. Hämarik, Projection methods and self-regularization in ill-posed problems, Soviet Mathematics 29 (1985), 1-20, in Russian. MR828379 (87m:65096)

33. G. Vainikko and A. Y. Veterennikov, Iteration procedures in ill-posed problems, Nauka, Moscow, 1986. MR859375 (88c:47019)

34. V.V. Vasin and A.L. Ageev, Ill-posed problems with a priori information, VSP, Zeist, 1995. MR $1374573(97 \mathrm{j}: 65100)$

Institute for Mathematics, University of Klagenfurt, Universitätssstrasse 65-67, A9020 Klagenfurt, Austria

E-mail address: barbara.kaltenbacher@aau.at

Institute for Stochastics and Applications, University of Stuttgart, PfaffenwaldRING 57, 70569 Stuttgart, Germany

E-mail address: jonas.offtermatt@mathematik.uni-stuttgart.de 